Volume 1, Issue 1, May - 2020: pp: 33-37_www.ejor.sohag-univ.edu.eg

Original article

\title{
FIXATION OF PILON FRACTURE \& COMMINUTED DISTAL TIBIAL FRACTURES WITH TRIANGULAR EXTERNAL FIXATOR
}

\author{
Hassan Ahmed, Mohamed Ali ${ }^{(*)}$, Moustafa Elsayed, Wael Salama, Shazly Mosa \\ Orthopedic Surgery dept., Faculty of Medicine, Sohag Univ., Sohag, Egypt \\ *E-mail: mohamedaliortho82gmail.com
}

Received 2/2/2020

Accepted 30/4/2020

\begin{abstract}
Treatment of Pilon fractures and comminuted distal tibia fractures represent a great challenge for surgeons. There are different methods of fixation with several complications including osteoarthritis and skin compromise. 20 adult patients with pilon fracture \& comminuted distal tibial fractures admitted in orthopedics and traumatology department of academic level 1 trauma center who were treated by triangular external fixator. Patients with pathological fracture, pre-existing ankle arthritis, previous fracture around the ankle and prolonged use of steroids were excluded. Clinical and functional evaluation was done using Olerud and Molander ankle score and radiological evaluation was done at 6 weeks, 3 months and 6 months follow up. The average age was $39(16-70)$ years old. 13 male patients. 16 patients of them had pilon fractures \& 4 of them had Extraarticular comminuted distal tibial fractures. 12 of them had simple fractures and 8 had compound fractures. The average time of healing was 3 months. 5 patients (25\%) showed excellent results, 9 patients (45\%) showed good results and 6 patients (30\%) showed fair results. Triangular external fixator is a good option in treatment of pilon and comminuted distal tibia fracture with good functional outcome and lower complication rate.
\end{abstract}

Keyword: Pilon, Distal tibia, Triangular external fixator

\section{Introduction}

Fractures of the distal tibia are considered one of the most difficult fractures to treat. The short distal segment and tight skin covering represent difficulties for the orthopaedic surgeon in choosing the appropriate fixation method [1]. The pilon fracture is an intra-articular group of these injuries. It represents 5-7\% of all tibial fractures [1-3]. It occurs due to high energy trauma and articular comminution is frequently present. The greatest challenge to the orthopaedic surgeon lies in the relatively tight soft tissue around the ankle. The conventional method of open reduction and fixation by plate and screws provides good outcomes only in the fractures with less severe, lower energy trauma [4-7]. The choice of treatment must take into account not only the stabilisation of the fracture, but also the management of the soft tissue condition which is a frequent cause of subsequent complications [8,9]. Timing of surgery is considered according to soft tissue condition. Anatomical reduction of intraarticular fractures is crucial to prevent the development of ankle osteoarthritis, which often results in a serious functional impairment [10,11]. Recent management of these fractures with external fixation with or without limited internal fixation has been used to avoid soft tissue and wound complications which are common with these fractures [12-14]. 


\section{Materials and Methods}

\subsection{Patients}

It is a retrospective study of 20 patients presented to our hospital by acute pilon fracture \& comminuted distal tibial fractures. All patients were treated at emergency operation by triangular external fixator. Patients with pathological fracture, preexisting ankle arthritis, previous fracture around the ankle and prolonged use of steroids were excluded. An informed written

\subsection{Operative technique}

Under spinal anesthesia, the patient in supine position on radiolucent table, tourniquet was applied to the proximal thigh. After sterilization of the limb, two schanzes applied to the tibia then one schanz centrally threaded to the calcaneus then connecting them by rods, fig. (1) and reduction is made by distraction (ligamentotaxis) between tibial and calcaneal shanzes, fig. (2). Fixat-

consent was obtained from all participants. The study was approved by our scientific $\&$ ethical committee. Clinical and functional evaluation was done using Olerud and Molander ankle score, tab. (1). A score 91-100 is considered excellent, 61-90 is good, 3160 is fair and $<30$ is poor. Radiological evaluation using X-ray was done at 6 weeks, 3 months and 6 months follow up.

ion of fibula was done 2 cases. Confirmation of reduction by flouroscopic anteroposterior, lateral and mortise views. Mobilization of knee joint was started on the second day. Weight bearing was proh-ibited till healing is evident radiologically and clinically after removal of the external fixator.

Table (1) Olerud and Molander ankle scoring system

\begin{tabular}{|l|l|c|}
\hline \multicolumn{1}{|c|}{ Pain } & \multicolumn{1}{|c|}{ None } & $\mathbf{2 5}$ \\
\hline & Minimal (according to the weather) & $\mathbf{2 0}$ \\
\hline & During sporting activities & $\mathbf{1 5}$ \\
\hline & Walking on even surfaces & $\mathbf{1 0}$ \\
\hline & Constant and severe & $\mathbf{0}$ \\
\hline Stiffness & None & $\mathbf{1 0}$ \\
\hline & When stretching & $\mathbf{5}$ \\
\hline & Constant & $\mathbf{0}$ \\
\hline Edema & None & $\mathbf{1 0}$ \\
\hline & Only the evening & $\mathbf{5}$ \\
\hline & Constant & $\mathbf{0}$ \\
\hline Stairs & No problems & $\mathbf{1 0}$ \\
\hline & Impaired & $\mathbf{5}$ \\
\hline & Impossible & $\mathbf{0}$ \\
\hline Sporting activities & Normal & $\mathbf{1 0}$ \\
\hline & Difficult & $\mathbf{5}$ \\
\hline & Impossible & $\mathbf{0}$ \\
\hline Supports & None & $\mathbf{1 0}$ \\
\hline & Taping/wrapping & $\mathbf{5}$ \\
\hline & Cane or crutches & $\mathbf{0}$ \\
\hline Daily life activities & Unchanged & $\mathbf{2 5}$ \\
\hline & Unchanged but slower & $\mathbf{2 0}$ \\
\hline & Less heavy work or part-time & $\mathbf{1 0}$ \\
\hline & Partially or totally impaired & $\mathbf{0}$ \\
\hline Total & & $\mathbf{1 0 0}$ \\
\hline
\end{tabular}




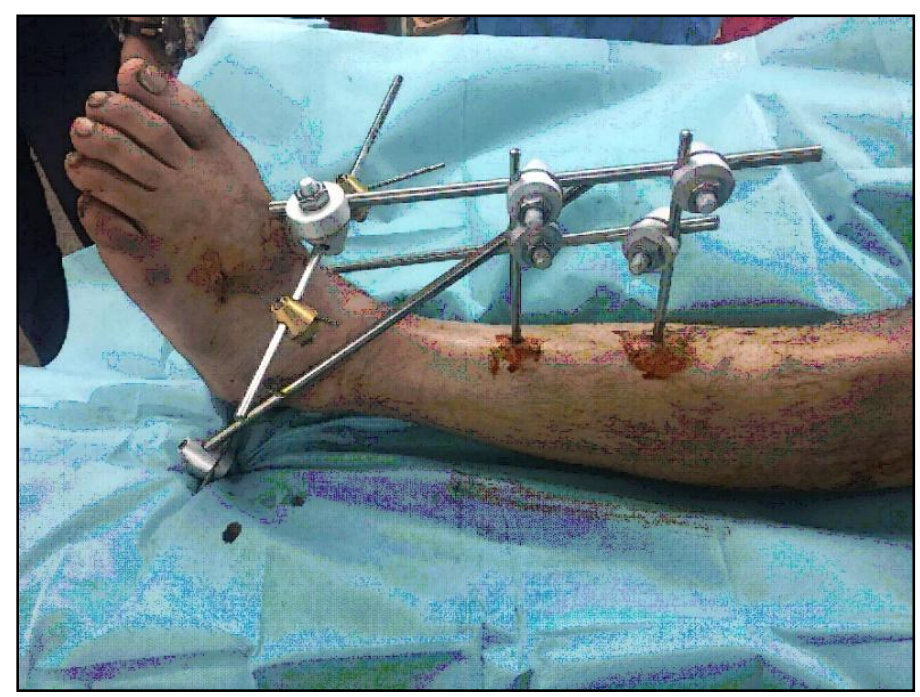

Figure (1) Shows triangular external fixator frame.
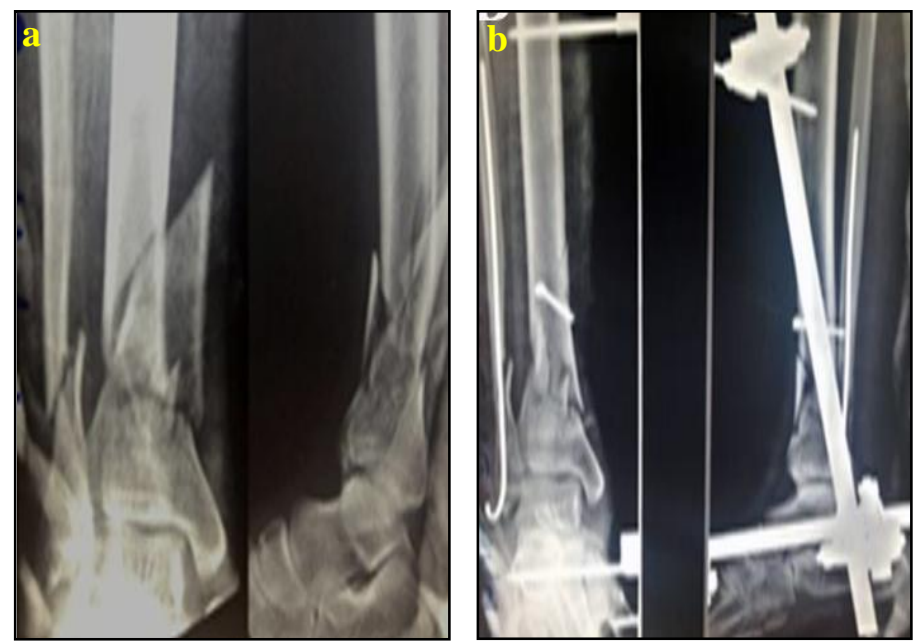

Figure (2) Shows reduction of comminuted distal tibia fracture by distraction using triangular external fixator $\underline{\mathbf{a}}$. preoperative X-ray, $\underline{\mathbf{b}}$. postoperative X-ray.

\section{Results}

This study included 20 patients (13 males). The average age was 39 (16-70) years old. Right side was affected in 5 cases. 16 patients of them had pilon fractures \& 4 of them had extraarticular comminuted distal tibial fractures. 12 of them had simple fractures and 8 had compound fractures. The average time of healing was 3 months. 5 patients $(25 \%)$ showed excellent results, 9 patients (45\%) showed good results and 6 patients $(30 \%)$ showed fair results. Supe-

\section{Discussion}

Distal tibial fractures are difficult to treat, not only due to the bony component, but also in terms of the soft tissue problem. In 1969, Ruedi and Allgower [15] reported rficial wound infection was observed in two cases and managed by debridement and I.V antibiotics. There were five cases presented with pin tract infection that recovered directly after device removal. Two cases show delayed union where the fracture healing takes about 9 months. One case complicated with varus deformity. Six cases complained from ankle joint arthritis. One case had a deep infection.

a $74 \%$ excellent and good functional result after they reviewed 84 pilon fractures treated with open reduction and internal fixation. While some authors $[4,5,16]$ pub- 
lished the same good results as Ruediand Allgower, others have reported less favorable outcomes with a high complication rate. Teeny and Wiss [7] reported $37 \%$ having deep infection and McFerran et al. [6] reported $40 \%$ of patients having a major complication. Wyrsch et al. [17] reported $16 \%$ amputations of patients having open reduction and internal fixation. These complications reflected the fact that extensive surgical dissection to achieve anatomical reduction jeopardizes the vascularity of bony fragments. These devascularized fragments will act as foci for infection. Also the bulky plate in this tight soft tissue envelope of the distal tibia also impaired wound healing. The incidence of skin slough and wound dehiscence was as high as $27 \%$ in the Teeny and Wiss series [7]. While anatomical reduction is necessary in the reconstruction of the articular surface, it is less important in the metaphyseal fragments. The recent use of external fixator with or without minimal internal fixation can solve both bony and soft tissue problems for better fracture healing. Reduction is achieved through ligament taxis and surgical dissection is minimized. Spanning external fixator device across the ankle joint had been used with promising result $[12,14$, 18]. Wyrsch et al. [17] compared between open reduction and internal fixation with external fixator with or without limited internal fixation in treating pilon fracture. The two groups showed similar results. However, the latter group was associated with fewer and less severe complications than internal fixation. The current study yielded favorable results concerning the use of this technique in treating pilon and comminuted distal tibia fractures with less severe complications which can be solved by minor interventions. The triangular configuration of the frame provides adequate stability for fracture healing rather than the conventional unilateral frame. This decreased the rate of nonunion which was reported in other studies.

\section{Conclusion}

Triangular external fixator is a good option in treatment of pilon and comminuted distal tibia fracture with good functional results and lower complication rate.

\section{References}

[1] Bedi, A., Le, T., Karunakar, M. (2006). Surgical treatment of nonarticular distal tibia fractures. J. Am Acad Orthop Surg. 14: 406-416.

[2] Lee, Y., Chen, S., Lin, J. et al. (2009). Surgical treatment of distal tibia fractures: A comparison of medial and lateral plating. Orthopedics. 32: 163.

[3] Topliss, C., Jackson, M., Atkins, R. (2005). Anatomy of pilon fractures of the distal tibia. J. Bone Joint Surg Br. 87: 692-697.

[4] Bourne, R. (1989). Pylon fractures of the distal tibia. Clin Orthop. 240: 42-46.

[5] Helfet, D., Koval, K., Pappas, J., et al. (1994). Intraarticular pilon fracture of the tibia. Clin Orthop. 298: 221228.

[6] McFerran, M., Smith, S., Boulas, H., et al. (1992). Complications encountered in the treatment of pilon fractures. J. Orthop Trauma. 6: 195-200.

[7] Teeny, S., Wiss, D. (1993). Open reduction and internal fixation of tibial plafond fractures. Clin Orthop 292: 108-117.

[8] Edwards, P. (1965). Fracture of the shaft of the tibia: 492 consecutive cases in adults, importance of soft tissue injury. Acta Orthop Scand. 76: 1-82.

[9] Ries, M., Meinhard, B. (1990). Medial external fixation with lateral plate internal fixation in metaphyseal tibia fractures: A report of eight cases associated with severe soft-tissue injury. Clin Orthop Relat Res. 256: 215-223.

[10] Borrelli, J., Ellis, E. (2002). Pilon fractures: Assessment and treatment. 
Orthop Clin North Am. 33: 231-245.

[11] Sirkin, M., Sanders, R., Di Pasquale, T., et al. (1999) A staged protocol for soft tissue management in the treatment of complex pilon fractures. $\boldsymbol{J}$. Orthop Trauma. 13: 78-84.

[12] Bonar, S., Marsh, J. (1993). Unilateral external fixation for severe pilon fractures. Foot and Ankle. 14:57-64.

[13] Bone, L., Stegemann, P., McNamara, K., et al. (1993). External fixation of severely comminuted and open tibial pilon fractures. Clin Orthop. 292: 101-107.

[14] Marsh, J., Bonar, S., Nepola, J., et al. (1995). Use of an articulated external fixator for fractures of the tibial plafond. J. Bone Joint Surg 77 A: 1498-1509.
[15] Ruedi, T., Allgower, M. (1969). Fractures of the lower end of the tibia into the ankle joint. Injury 1: 92-99,

[16] Kellam, J., Waddell, J. (1979). Fractures of the distal metaphysis with intraarticular extension: The distal tibia explosion fracture. J. Trauma. 19: 593-601,

[17] Wyrsch, B., McFerran, M., McAndrew, M., et al. (1996). Operative treatment of fractures of the tibial plafond. A randomized, prospective study. $\boldsymbol{J}$. Bone Joint Surg. 78 A: 1646-1657,

[18] Tornetta, P., Weiner, L., Bergman, M, et al. (1993). Pilon fractures: Treatment with combined internal and external fixation. J. Orthop Trauma . 7: 489-496. 